\title{
Influência do escore corporal no perfil lipídico de cachorros-do-mato (Cerdocyon thous) sob os cuidados humanos
}

HIPPÓLITO, Alícia Giolo1; TESTA, Carolyne Assis Eigenheer Pinke1; SANTOS, Gisele Junqueira dos; FERRO, Barbara Sardela2; SILVA, Diogo Cristo da Silva e ${ }^{2}$; MELCHERT, Alessandra ${ }^{3}$

\footnotetext{
1 Doutorandos do Programa de Pós-Graduação de Animais Selvagens - UNESP, Botucatu

2 Mestrandos do Programa de Pós-Graduação de Animais Selvagens - UNESP, Botucatu

3 Docentes da UNESP, Botucatu

E-mail: aliciamedvet@gmail.com
}

RESUMO: A obesidade é uma desordem nutricional complexa e multifatorial que alguns animais selvagens podem desenvolver ao longo de sua vida em cativeiro. O estudo comparou o perfil lipídico de cachorros-do-mato (Cerdocyon thous) em escore de condição corporal normal com o de animais em sobrepeso. Os animais eram hígidos e mantidos sob cuidados humanos sem enriquecimento ambiental. Apesar de poucos cachorros-do-mato ( $C$. thous) terem apresentado aumento de colesterol e triglicerídeos, não exibiram diferença estatística quando comparado os grupos de escore ideal e sobrepeso. Mais estudos devem ser realizados para verificar a influência do ganho de peso corporal sobre o perfil lipídico da espécie, mas o enriquecimento ambiental deve ser estimulado, pois proporciona aumento da atividade física, menor tendência do animal à obesidade, melhoria da qualidade de vida e bem-estar.

Palavras-chave: colesterol, obesidade, selvagens, sobrepeso, triglicerídeos.

\section{INTRODUÇÃO}

A hiperlipidemia pode ser definida como aumento de triglicerídeos (hipertrigliceridemia), colesterol (hipercolesterolemia) ou ambos. Pode ser consequente de defeito primário no metabolismo de lipoproteínas ou decorrente de uma doença sistêmica subjacente e, na grande maioria dos casos, está presente em cães domésticos com sobrepeso (BRUNETTO et al., 2011). A obesidade é uma desordem nutricional complexa e multifatorial, e exige manejo adequado (LINDER; MULLER, 2014). Muitos animais selvagens podem desenvolver essa patologia quando estão em cativeiro. As causas incluem maior disponibilidade de alimento, baixo nível de atividades e fatores predisponentes genéticos (RANDIN et al., 2009).A manutenção de animais selvagens em cativeiro, hoje versa sob uma nova ciência, propondo bem $\urcorner$ estar e à adaptação dos animais a ambientes artificiais (ecotização de recintos), proporcionando atividades que visam diminuir 0 estresse $e \quad 0$ vazio ocupacional desses animais (FILIPPE; ADANIA, 2014), porém ainda há locais que não dispõe tal estrutura. Esse estudo tem como objetivo comparar o perfil lipêmico de cachorros-do-mato de cativeiro com escore de condição corporal normal e animais em sobrepeso.

\section{MATERIAL E MÉTODOS}

Foram utilizados 9 cachorros-domato (C. thous) hígidos, (oito machos e 
uma fêmea) pertencentes ao Centro de Medicina e Pesquisa de Animais Selvagens (CEMPAS) e as amostras foram processadas no Laboratório Clínico do Hospital Veterinário da Faculdade de Medicina Veterinária e Zootecnia - FMVZ, UNESP/BotucatuSP. O trabalho foi aprovado pela Comissão de Ética no Uso de Animais (CEUA), protocolo 177/2015, e SISBio 51767-1. Os animais eram alimentados com frutas, ovo cozido, camundongo ou rato de biotério recém-abatidos e ração comercial de cães. As frutas compunham aproximadamente $40 \%$ da dieta, composta basicamente de banana, mamão, maçã, manga e melancia, de acordo com a disponibilidade. Permaneceram no CEMPAS pelo menos 6 meses com essa alimentação antes de iniciar essa avaliação. $O$ escore corporal foi avaliado de acordo com a escala de 1 a 5 , sendo 1 caquético; 2 magro; 3 ideal; 4 sobrepeso e 5 obeso. Os cachorros foram separados em dois grupos, normais $(n=4)$ e sobrepeso $(n=5)$. Os animais foram submetidos ao jejum hídrico e alimentar $(6$ e $12 \mathrm{~h}$, respectivamente). Foram contidos fisicamente com auxílio de um puçá e aplicada por via intramuscular a anestesia dissociativa composta por cetamina e midazolam nas doses de $10 \mathrm{mg} / \mathrm{kg}$ e $1 \mathrm{mg} / \mathrm{kg}$, respectivamente. Após a indução anestésica, o animal foi transferido para 0 ambulatório do CEMPAS para avaliação. O sangue foi coletado da veia jugular, após prévia antissepsia com clorexidine alcóolico e álcool $70^{\circ}$, utilizando seringa de $3 \mathrm{ml} \mathrm{e}$ agulha $25 \times 7$, acondicionado em tubo estéril sem anticoagulante e posteriormente centrifugado para separação do soro. Foram avaliados o colesterol total (CT) e triglicerídeos (TG), pelo método colorimétrico enzimático, utilizando o Kit Labtest ${ }^{\circledR}$, dosados em analisador semiautomático (Quick Lab II - Drake ${ }^{\circledR}$ ).

\section{RESULTADOS E DISCUSSÃO}

Dos nove animais em cativeiro, cinco apresentaram condição de sobrepeso. O perfil lipídico está descrito nas tabelas 1 e 2, de acordo com o escore corporal.

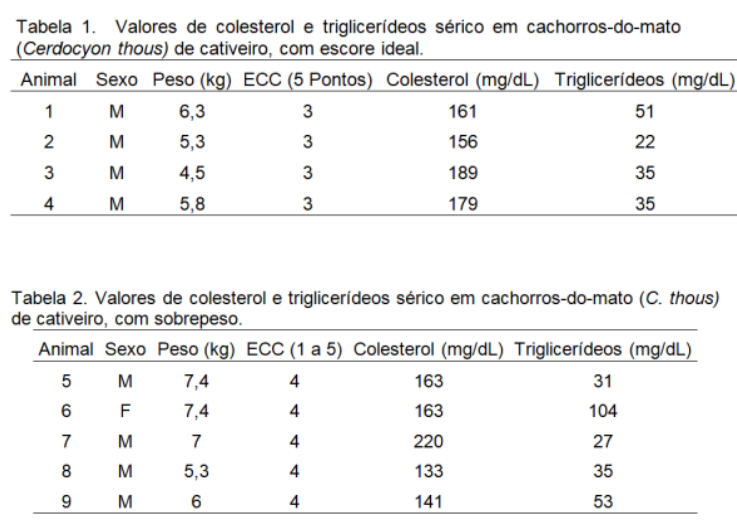

O cachorro do mato é uma espécie de canídeo silvestre, onívoro, com peso médio entre 4,5 a $8,5 \mathrm{~kg}$ (BERTA, 1982), mas essa informação não é suficiente para avaliar o escore corporal dos indivíduos de cativeiro. Apesar de alguns indivíduos exibirem excesso de peso, observado pelo escore 4 e a presença de colesterol e triglicerídeos aumentados, o teste $\mathrm{T}$ de Student não pareado, e não apresentou diferença entre os dois grupos estudados $(p<0.05)$. Cães obesos apresentam distúrbios como a dislipidemia (VERKEST, 2014). Entretanto, MARTINS (2012) observaram que apenas $13,3 \%$ dentre 30 cães domésticos obesos apresentaram elevação do colesterol sérico, similar ao presente estudo. Devem ser realizados mais estudos com esse objetivo, para afirmar com segurança o perfil lipídico relacionado ao excesso de peso nessa espécie.

\section{CONCLUSÃO}

Embora não se tenha encontrado diferença do perfil lipídico de cachorros do mato em sobrepeso, observou-se 
alta taxa de sobrepeso, sendo imprescindível a aplicação de enriquecimento ambiental nos recintos dos animais selvagens, estimulando maior grau de atividade física, melhor qualidade de vida e bem-estar para os animais sob cuidados humanos.

\section{AGRADECIMENTOS}

Os autores agradecem ao Centro de Medicina e Pesquisa de Animais Selvagens - CEMPAS, pelos dados dos animais.

\section{REFERÊNCIAS}

BERTA A. Cerdocyon thous. Mammalian species, v.847, p 1-4. 1982.

FELIPPE, P. A. N.; ADANIA, C. H.; Conservação e Bem estar Animal In: In: Cubas, Z. S.; Silva, J. C. R.; Catão-Dias, J. L.; Tratado de Animais Selvagens; 2014.

BRUNETTO, M. A.; NOGUEIRA, S.; SÁ, F. C.; PEIXOTO, M.; VASCONCELLOS, R. S.; FERRAUDO, A. J.; CARCIOFI, A. C; Correspondência entre obesidade e hiperlipidemia em cães; Ciência Rural, Santa Maria, v.41, n.2, p.266-271, fev, 2011.

LINDER, D.; MUELLER, M. Pet Obesity Management. Vet Clin North Amer: Small Animal Practice, v. 44, n. 4, p.789806, 2014.

MARTINS, C. R.; Perfil hematológico e bioquímico de cães (Canis familiaris) obesos e idosos. (Dissertação) Universidade Federal de Uberlândia Faculdade de Medicina Veterinária, Programa de pós-graduação em Ciências Veterinárias, 2012.

RANDIN, M. J.; SHARKEY, L. C.; HOLYCROSS, B. J.. Adipokines: a review of biological and analytical principles and an update in dogs, cats, and horses. Vet Clin Pathol, v. 38, n. 2, p.136-156, 2009.

VERKEST, K.R. Is the metabolic syndrome a useful clinical concept in dogs? A review of the evidence. Vet J; v.199, n.1, p.24-30, 2014.

Agradecemos ao apoio da Fundação Araucária e da Coordenação de Aperfeiçoamento de Pessoal de Nível Superior - CAPES na realização do $3^{\circ}$ Workshop de Nutrição de Animais Selvagens.
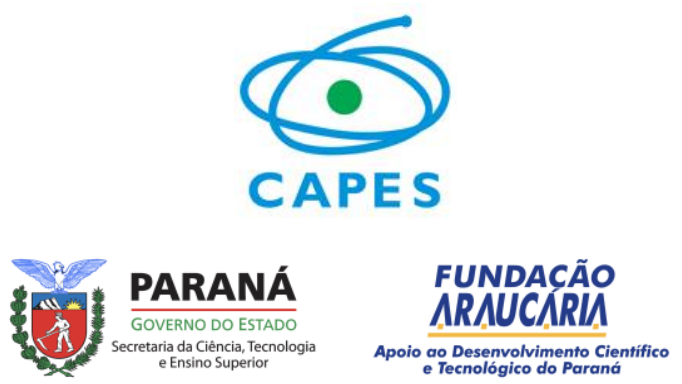